\title{
Recovery of Liver Motion and Deformation Due to Respiration Using Laparoscopic Freehand 3D Ultrasound System
}

\author{
Masahiko Nakamoto ${ }^{1}$, Hiroaki Hirayama ${ }^{1}$, Yoshinobu Sato ${ }^{1}$, Kozo Konishi $^{2}$, \\ Yoshihiro Kakeji ${ }^{2}$, Makoto Hashizume ${ }^{2}$, and Shinichi Tamura ${ }^{1}$ \\ ${ }^{1}$ Division of Image Analysis, Graduate School of Medicine, Osaka University, Japan \\ ${ }^{2}$ Graduate School of Medical Sciences, Kyushu University, Japan
}

\begin{abstract}
This paper describes a rapid method for intraoperative recovery of liver motion and deformation due to respiration by using a laparoscopic freehand 3D ultrasound (US) system. Using the proposed method, 3D US images of the liver can be extended to 4D US images by acquiring additional several sequences of $2 \mathrm{D}$ US images during a couple of respiration cycles. Time-varying 2D US images are acquired on several sagittal image planes and their $3 \mathrm{D}$ positions and orientations are measured using a laparoscopic ultrasound probe to which a miniature magnetic 3D position sensor is attached. During the acquisition, the probe is assumed to move together with the liver surface. In-plane 2D deformation fields and respiratory phase are estimated from the timevarying $2 \mathrm{D}$ US images, and then the time-varying $3 \mathrm{D}$ deformation fields on the sagittal image planes are obtained by combining 3D positions and orientations of the image planes. The time-varying $3 \mathrm{D}$ deformation field of the volume is obtained by interpolating the $3 \mathrm{D}$ deformation fields estimated on several planes. The proposed method was evaluated by in vivo experiments using a pig liver.
\end{abstract}

\section{Introduction}

In laparoscopic liver surgery, 3D ultrasound (3D US) is a useful modality for intraoperative 3D imaging of internal structures of the liver such as vessels or tumors due to its realtime and non-invasive nature. Aiming at safe and accurate surgery, the internal structures can be virtually seen through by superimposing the 3D US onto the real laparoscope view. Motion and deformation of the liver due to respiration is a major problem in maintaining accurate superimposition. Although breath holding during superimposition is often performed, it not only disturbs the smooth operation but may affect the patient condition if it is performed many times. Olbrich et al. proposed a gating method for visualizing the superimposition only at the expiration phase, which is regarded as the most stable phase in the respiratory cycle [1], However, its visualization is not continuous but intermittent. On the other hand, studies on recovery of liver motion from preoperative MR images have been reported [2] 3] 4]. There are the following disadvantages in intraoperative use of these methods: (1) Preoperative 
images may not capture actual intraoperative dynamic motion of the liver in the laparoscopic setup in which the abdominal cavity is filled with air. (2) Acquisition of time-varying 3D MR images in acceptable spatiotemporal resolution is time-consuming.

In this paper, we describe a method for intraoperative recovery of respiratory motion and deformation of the liver using a laparoscopic freehand 3D US system for the purpose of augmented reality visualization of 4D US. Advantages of the proposed method are as follows:

- The data acquisition protocol is simple and rapid so that it can be performed intreoperatively. Acquisition of several cross-sectional time-varying 2D US images is only required in addition to conventional 3D US image acquisition using a freehand probe.

- Intraoperative dynamic motion and deformation of the liver in the laparoscopic setup are recovered.

The proposed method utilizes the assumptions on the characteristics of the liver motion indicated in the previous work [2]. Its main component exists in the cranio-caudal $(5-25 \mathrm{~mm})$ and anterior-posterior $(1-12 \mathrm{~mm})$ directions, and the component in the left-right direction is sufficiently small (1-3 mm) 2]. That is, we assume that the nonrigid deformation mainly exists in the sagittal planes and is negligible in the left-right direction (although rigid motion component across the sagittal planes is incorporated in our method). In addition, we assume that the liver motion as periodical because the patient is under general anesthesia and the breathing is controlled by the respirator. The proposed method combines rigid $3 \mathrm{D}$ motions of the image planes corresponding to the US probe motion and time-varying in-plane 2D deformation fields estimated from time-varying 2D US images acquired at several (quasi-)sagittal planes to recover the $4 \mathrm{D}$ deformation field of the liver. Since the laparoscopic US probe is in contact with the liver surface during the US image acquisition, we assume that the US probe motion corresponds to the liver surface motion with moderate contact pressure. We perform animal experiments to evaluate and demonstrate feasibility of the proposed method.

\section{Methods}

\subsection{System Overview and Data Acquisition}

We employ a freehand 3D ultrasound system described in [5]. The position and orientation of the probe tip are measured using a miniature magnetic tracker attached to the tip of the ultrasound probe (Fig. 11). Spatial relationship between the tracker and the US image is determined by preoperative calibration. Magnetic field distortion caused by metallic objects is corrected in a rapid manner by using the method described in [6] just before US image acquisition.

Time-varying 2D US image sequences of several (quasi-)sagittal planes as well as their positions and orientations are acquired during two or more respiratory 
cycles. We assume that the probe tip is put directly on the liver surface with moderate contact pressure so that it moves together with the liver surface. The time-varying data set $D_{i}$ of $i$-th sagittal plane is defined as

$$
D_{i}=\left\{\left(I_{i}(t), T_{i}(t), R_{i}(t)\right) \mid t=1, \ldots, N_{i}\right\}
$$

where $I_{i}(t)$ is time-varying $2 \mathrm{D}$ US images, and $T_{i}(t)$ and $R_{i}(t)$ are positions and orientations of the US images, respectively. $t$ denotes a frame number and $N_{i}$ is the total number of the acquired frames.
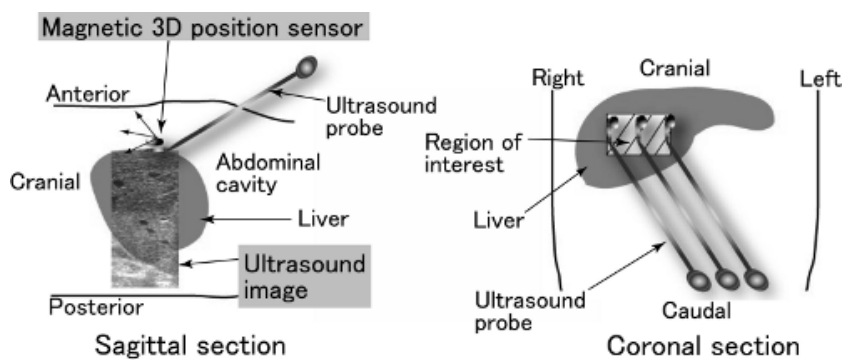

Fig. 1. Intraoperative position measurement and laparoscopic ultrasound image acquisition of the liver

\subsection{Estimation of Respiratory Cycle}

Temporal registration among acquired time-varying data sets is necessary. To do so, the reference points of time in the respiratory cycle for time-varying data sets of different sagittal planes are determined. As reference points, we determine the start and end points of deformation as well as the inspiration and expiration points for each time-varying data set by analyzing the positions of US image planes and time-varying similarities between temporally adjacent US images. Normalized cross correlation (NCC) is used as a similarity measure, which is considered to be in inverse proportion to the amount of deformation. Figure 2 shows an example of time-varying similarities and positions of the image plane. The respiratory cycle is divided into static and dynamic phases based on the magnitude of similarity. In the static phase, the similarity has a nearly constant and large value. The start point of deformation is determined as a point where the similarity begins to decrease (point D in Fig. 2). The end point of deformation is also determined as a point where the similarity returns to the large value (point B in Fig. 2). Using points D and B, the dynamic and static phases are divided. The inspiration point is determined as the peak point during the dynamic phase (point A denoted by circles in Fig. 2), and is used as a reference point for temporal registration since it can be localized stably and accurately. The point where the change in both US images and the position is the smallest is determined as the expiration point (point C in Fig. 2), 2D US image at the expiration point is used as a reference image for nonrigid registration described in the next subsection since it is regarded as the most stable state. 


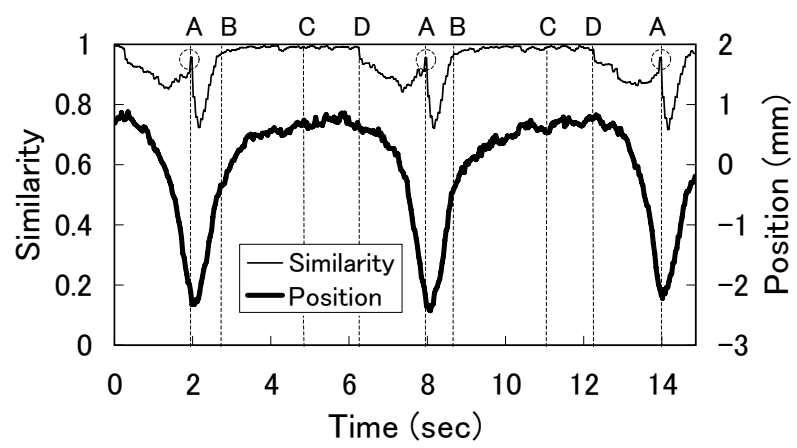

Fig. 2. Analysis of respiratory cycle. A: Inspiration point. B: Start point of deformation. C: Expiration point. D: End point of deformation.

We confirmed that the above four reference points could be stably localized by 1D signal analysis of time-varying similarities and positions shown in Fig. 2 (although the details of the methods are not described here). The respiratory cycle length is determined as the interval between adjacent inspiration points.

\subsection{Estimation of In-Plane Deformation}

To estimate time-varying in-plane deformation fields from the US image sequences, we perform intensity-based non-rigid registration [7. NCC is used as a similarity measure. The difference between temporally adjacent $k$-th and $(k+1)$ th deformations is regarded as small since respiratory motion and deformation are smooth and continuous. Therefore, estimated $k$-th deformation is used as an initial deformation for the estimation of $(k+1)$-th deformation. $k$-th $2 \mathrm{D}$ deformation field $\mathbf{m}_{i}(k)$ is defined as $\mathbf{m}_{i}(k)$ maximizing

$$
N C C\left(F\left(I_{i}\left(t_{e, i}\right), \mathbf{m}_{i}(k)\right), I_{i}\left(t_{e, i}+k-1\right)\right),
$$

where $N C C(I, J)$ denotes NCC of images $I$ and $J, F(I, \mathbf{m})$ denotes the deformed image of $I$ by deformation field $\mathbf{m}$, and $t_{e}$-th frame corresponds to the expiration point (point $\mathrm{C}$ in Fig. 2). To accurately estimate the deformation of the liver, the liver region is manually segmented beforehand only in the $t_{e}$-th frame, which is not time-consuming since only one short curve is specified at the bottom of the liver.

\subsection{Recovery of 4D Motion and Deformation}

The recovery processes of 4D liver motion and deformation consist of three stages. Firstly, temporal registration among time-varying data sets is performed by aligning the inspiration points (point A in Fig. 2), and then the frame offset $\gamma_{i}$ of each time-varying data set is obtained. The normalized frame number $t^{\prime}=t_{i}-\gamma_{i}$ represents the same respiratory phase among the time-varying data sets. Secondly, by combining the position and orientation of a US image 
$\left(T_{i}\left(t^{\prime}\right), R_{i}\left(t^{\prime}\right)\right)$ and deformation field $\mathbf{m}_{i}\left(t^{\prime}\right), 3 \mathrm{D}$ deformation vector $\mathbf{m}_{i}^{\prime}\left(t^{\prime}\right)$ at an arbitrary position $\mathbf{p}$ on the US image plane is estimated as

$$
\mathbf{m}_{i}^{\prime}\left(\mathbf{p}, t^{\prime}\right)=R_{i}\left(t^{\prime}\right) \mathbf{m}_{i}\left(\mathbf{p}, t^{\prime}\right)+\left(R_{i}\left(t^{\prime}\right)-R_{i}\left(t_{0}^{\prime}\right)\right) \mathbf{p}+\left(T_{i}\left(t^{\prime}\right)-T_{i}\left(t_{0}^{\prime}\right)\right) .
$$

Finally, the deformation vector at an arbitrary 3D position in the whole volume covering the 3D US image is obtained by performing interpolation of the 3D deformation fields on the 2D US image planes. In order to interpolate the 3D vectors sampled in a scattered nature due to freehand image acquisition, we employ a multilevel B-spline fitting method [8].

\section{Experimental Results}

\subsection{Experimental Conditions}

We performed in vivo experiments using a pig in the laparoscopic environment to evaluate the proposed method. The pig was under general anesthesia and its breathing was controlled by the respirator. The ventilation volume was $400 \mathrm{cc}$. SSD-5500 (ALOKA Co., Japan) was employed as an ultrasound system. The field of view of the US images was $27 \times 56 \mathrm{~mm}^{2}$, and the frame rate was 30 frames per second. microBIRD (Ascension Technology Co., Burlington, VT) was employed to magnetically track the US probe tip. Polaris (Northern Digital Inc., Waterloo, Ontario, Canada) was employed for correction of the magnetic field distortion of microBIRD using a magneto-optic hybrid tracker [6].

We acquired two data sets around the porta hepatic of the pig, which covered the volume of $27 \times 56 \times 60 \mathrm{~mm}^{3}$ and $27 \times 56 \times 90 \mathrm{~mm}^{3}$, respectively. These data sets consisted of six and seven 2D-US sagittal planes of average intervals of $12.6 \mathrm{~mm}$ and $15.7 \mathrm{~mm}$, respectively.

\subsection{Recovered Motion and Deformation}

Figure 3 shows the motion and deformation of the pig liver recovered from the data set. The estimated respiratory cycle length was 6.0 seconds. The amount of recovered motion was the largest in the cranio-caudal, next largest in the anterior-posterior, and the smallest in the left-right directions. It was maximum at the inspiration point in all the three directions.

The recovered $4 \mathrm{D}$ deformation fields were combined with the 3D US images of the same regions as those covered by the data sets acquired for recovery of the motion and deformation in order to reconstruct the 4D US images. Figure 4 shows the 3D liver vessel model reconstructed from the 3D US images and the US image frames of sagittal planes used for recovery of the motion and deformation. The 3D US images were acquired at the expiration phase during breath holding. Figure 5 shows the $4 \mathrm{D}$ liver vessel model reconstructed by combining the 3D liver vessel model and the recovered 4D deformation field. Distinctive motion was observed from the lateral view, where the vessels moved up and down along the different paths during the expiration and inspiration phases, respectively. 


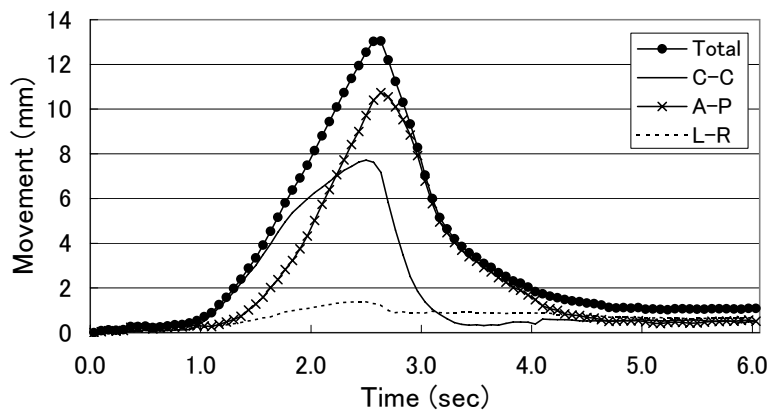

Fig. 3. Magnitude of recovered motion of the liver along the body axis (Data set 1). CC: cranio-caudal movement. A-P: anterior-posterior movement. L-R: lateral movement.
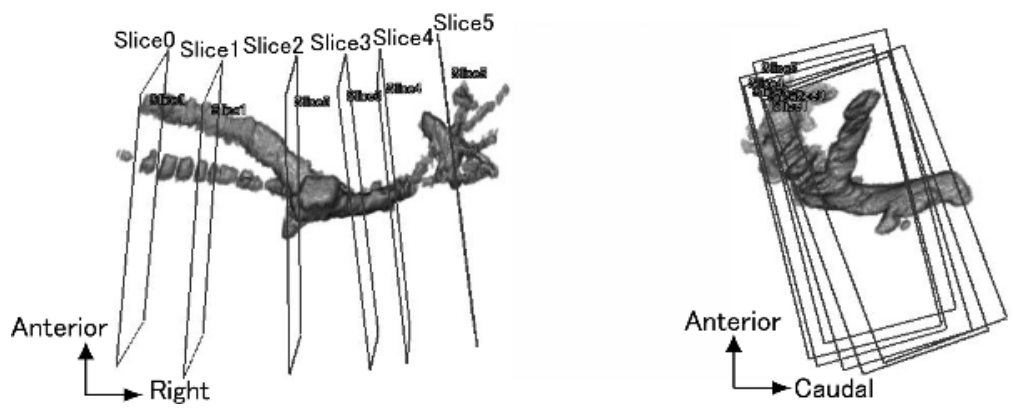

Fig. 4. 3D US liver vessel model and US image frames of sagittal planes (Data set 1). $\mathrm{C}-\mathrm{C}$ : the cranio-caudal axis. A-P: the anterior-posterior axis. L-R: the lateral axis.

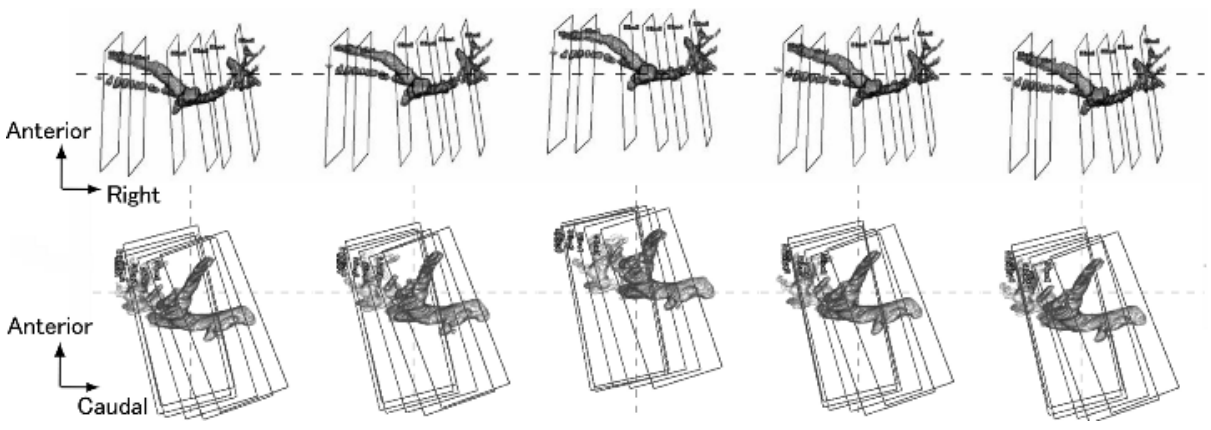

Fig. 5. Reconstructed $4 \mathrm{D}$ liver vessel model (Data set 1)

\subsection{Accuracy Evaluation of Recovered 4D Deformation Field}

To evaluate the effects of intervals of sagittal planes on the accuracy, we performed leave- $N$-out cross validation. Recovery of the deformation fields was performed by removing selected $N$ planes and then the deformation fields on the 
$N$ planes were used for validation by comparing them with the recovered deformation field of the whole volume.

The validation planes were selected based on three conditions; (a) a single plane, (b) every one plane, and (c) every two of three planes. The average interval was the smallest in condition (a) and the largest in condition (c). The error $E_{i, t}$ was defined as

$$
E_{i, t}=\sqrt{\frac{\sum_{x, y, z \in R_{i}}|\operatorname{Val}(x, y, z, t)-\operatorname{Est}(x, y, z, t)|^{2}}{n}},
$$

where $n=\sum_{x, y, z \in R_{i}} 1$, and $R_{i}$ was the point set on the selected planes. $\operatorname{Val}(x, y, z, t)$ and $\operatorname{Est}(x, y, z, t)$ were original and recovered deformation vectors at position $(x, y, z)$ and time $t$, respectively. Here, the original deformation vectors means the $3 \mathrm{D}$ deformation field estimated in the second step of 2.4.

Table 1 summarizes the results. The error increased during the period from the expiration point to the inspiration point and then decreased to the next expiration point. The maximum error was observed around the inspiration point. The magnitude of error along time was correlated to that of respiratory motion, and the error remained $1.0-1.5 \mathrm{~mm}$ at the end of the cycle. The maximum error under condition (b) during one cycle was comparable to condition (a), and it was around $2.0 \mathrm{~mm}$ (Table1).

Table 1. Results on accuracy evaluation for recovery of deformation vectors in whole volume

\begin{tabular}{|l|l|c|c|c|}
\hline & Condition & (a) & (b) & $(\mathrm{c})$ \\
\hline \multirow{3}{*}{ Data set 1} & Average interval $(\mathrm{mm})$ & 15.8 & 25.2 & 31.3 \\
\cline { 2 - 5 } & Maximum error $(\mathrm{mm})$ & 1.9 & 1.8 & 2.2 \\
\cline { 2 - 5 } & Average error $(\mathrm{mm})$ & 1.3 & 1.2 & 1.2 \\
\hline \multirow{3}{*}{ Data set 2} & Average interval $(\mathrm{mm})$ & 15.7 & 18.8 & 42.2 \\
\cline { 2 - 5 } & Maximum error $(\mathrm{mm})$ & 2.4 & 2.3 & 2.7 \\
\cline { 2 - 5 } & Average error $(\mathrm{mm})$ & 1.0 & 1.0 & 1.1 \\
\hline
\end{tabular}

\section{Discussion and Conclusions}

We have described an intraoperative recovery method of respiratory motion and deformation of the liver using a laparoscopic freehand 3D-US system. We confirmed that plausible motion and deformation of the pig liver were recovered from several time-varying 2D US images whose 3D positions and orientations were measured. The characteristics of the recovered motion were qualitatively consistent with those reported previously. The results of the cross validation to evaluate the interpolation accuracy showed the estimation error of the deformation field was around $2 \mathrm{~mm}$ even at the inspiration point using additional acquisition of three 2D US images during a couple of respiratory cycles for the volume of around $27 \times 56 \times 60-90 \mathrm{~mm}^{3}$. The additional image acquisition time was two or three minutes. Therefore, the 3D US images of the liver can be 
extended to the 4D US images by spending a little time on acquiring additional data sets. Computation time was around 30 minutes in current implementation, but it might be significantly reduced by introducing GPU-based technologies.

To recover $3 \mathrm{D}$ deformation field from $2 \mathrm{D}$ deformation fields on several sagittal planes, we assume that nonrigid deformation in the lateral direction is negligible although rigid translation in the lateral direction was incorporated as motion of the whole image frame. If large deformation in the lateral direction occurs, the estimation of in-plane deformation would not be accurate. However, the recovered motion in the lateral direction was less than $2 \mathrm{~mm}$ in the recovered deformation field. We validated the in-plane deformation using several localizable feature points, and its accuracy was less than $1 \mathrm{~mm}$ in average. Another assumption was that the liver motion as periodical. We also validated that the repeatability of the pig liver motion was much higher in the laparoscopic environment than the open surgery environment. Although there is concern that a trocar may restrict the US probe movement, the US probe tip could be moved smoothly together with the liver surface during several respiratory cycles under laparoscope. In addition, all of the time-varying 2D US images could be adjusted almost parallel to the sagittal plane. We conclude that the potential usefulness of the proposed method was demonstrated based on the above considerations. Future work will include accuracy evaluation using markers embedded in the pig liver.

\section{References}

1. Olbrich, B., Traub, J., Wiesner, S., et al.: Respiratory motion analysis: Towards gated augmentation of the liver. In: Proceedings of Computer Assisted Radiology and Surgery (CARS 2005), Berlin, Germany (2005) 248-253

2. Rohlfing, T., Maurer Jr., C.R., O'Dell, W.G., Zhong, J.: Modeling liver motion and deformation during the respiratory cycle using intensity-based nonrigid registration of gated MR images. Medical Physics 31 (2004) 427-432

3. Penney, G., Blackall, J., Hawkes, D.J.: Registration of freehand 3D ultrasound and magnetic resonance liver images. Medical Image Analysis 8 (2004) 81-91

4. von Siebenthal, M., Cattin, P., Gamper, U., et al.: 4D MR imaging using internal respiratory gating. In: Lecture Notes in Computer Science, 3750 (Proc. MICCAI 2005, Part II), Palm Springs, CA, USA (2005) 336-343

5. Anonymous: 3D ultrasound system using a magneto-optic hybrid tracker for augmented reality visualization in laparoscopic liver surgery. In: Lecture Notes in Computer Science, 2489 (Proc. MICCAI 2002, Part II), Tokyo, Japan (2002) 148-155

6. Anonymous: A rapid method for magnetic tracker calibration using a magneto-optic hybrid tracker. In: Lecture Notes in Computer Science, 2879, (Proc. MICCAI 2003, Part II), Montreal, Canada (2003) 285-293

7. Rueckert, D., Sonoda, L.I., Hayes, C., et al.: Nonrigid registration using free-form deformations: Application to breast MR images. IEEE Trans. Med. Imag. 18 (1999) $712-721$

8. Lee, S., Wolberg, G., Shin, S.Y.: Scattered data interpolation with multilevel Bsplines. IEEE Trans. Visualization and Computer Graphics 3 (1997) 228-244 